\title{
Diseño de una herramienta guía para la implementación de Lean en laboratorios de educación superior
}

\author{
Diana Carolina Bojacá Torres** \\ Ivonne Angélica Castiblanco Jiménez ${ }^{* * *}$ \\ Jairo Raúl Chacón Vargas ${ }^{* * * *}$
}

Recibido: 09/07/2019 - Aceptado: 25/09/2019

https://doi.org/10.22395/rium.v19n36a7

\begin{abstract}
Resumen
El artículo propone una herramienta guía para la implementación de Lean en laboratorios de educación superior, la cual se aplicó en el laboratorio de suelos y pavimentos de la Escuela Colombiana de Ingeniería Julio Garavito. Con esta herramienta, que incorpora de manera detallada los pasos para su aplicación, se busca reducir desperdicios en estos ambientes tales como un entrenamiento insuficiente, falta de supervisión, experiencia, medidas de rendimiento sistemáticas, responsabilidad, disponibilidad de materiales, y en general, la ausencia de una disposición sistemática del laboratorio y del equipo. Los resultados alcanzados presentan mejoras importantes a corto y mediano plazo en los procesos de registro y préstamo de equipos y herramientas. El caso analizado evidencia resultados positivos, que tienen el potencial de servir de ejemplo para otras organizaciones del sector de la educación que deseen adaptar Lean con sus herramientas como una alternativa viable, práctica y de bajo costo.
\end{abstract}

Palabras clave: lean manufacturing; lean en laboratorios; lean en educación superior; kaizen; mejora continua.

* $\quad$ El artículo es derivado de un proyecto de grado titulado Lean y su aplicación en el laboratorio de suelos y pavimentos de la Escuela Colombiana de Ingeniería, ejecutado entre agosto y diciembre de 2017 desde el programa de Ingeniería Industrial en la Escuela Colombiana de Ingeniería Julio Garavito. Financiación Escuela Colombiana de Ingeniería Julio Garavito.

** Ingeniera civil, ingeniera industrial, profesora catedrática. Facultad de Ingeniería Civil, Escuela Colombiana de Ingeniería Julio Garavito, Bogotá, Colombia. Correo electrónico: diana.bojaca@escuelaing.edu.co Orcid: https://orcid.org/0000-0002-1930-5053

*** Ingeniera electrónica, máster en Mecatrónica, máster en Automatización Industrial. Researcher, Politecnico di Torino, Italia. Correo electrónico: ivonne.castiblanco@polito.it Orcid: https://orcid.org/0000-0001-5866-078X

**** Ingeniero industrial, doctor en Ingeniería, profesor asociado, programa de Ingeniería Industrial, Escuela Colombiana de Ingeniería Julio Garavito, Bogotá, Colombia. Correo electrónico: jairo.chacon@escuelaing.edu. co Orcid: https://orcid.org/000-0002-9766-369X 


\title{
Design of a Framework for Lean Implementation in Higher Education Labs
}

\begin{abstract}
This article proposes a framework for the implementation of Lean Laboratories in higher education laboratories, which was applied in the soil and pavements laboratory of the Escuela Colombiana de Ingeniería Julio Garavito. With this framework, which incorporates in a detailed manner the steps for its application, the project aims towards decreasing the residues in these environments such as insufficient training, lack of supervision and experience, systematic performance measures, responsibility, materials availability, and generally, the absence of a systematic disposition of the laboratory and the equipment. The results achieved show important improvements in the short and medium-term in the registry and lending processes of tools and equipment. The analyzed case displays positive results with the potential of being exemplary for other organizations in the sector with aims towards implementation Lean and its tools as a functional, practical, and low-cost alternative.
\end{abstract}

Keywords: Lean manufacturing; Lean in laboratories; Lean in higher education; Kaizen; continuous improvement.

\section{Desenho de uma ferramenta guia para a implementação de Lean em laboratórios de ensino superior}

\begin{abstract}
Resumo
Este artigo apresenta uma ferramenta guia para a implementação de Lean em laboratórios de ensino superior, que já foi aplicada no laboratório de solos e pavimentos da Escuela Colombiana de Ingeniería Julio Garavito. Com essa ferramenta, que incorpora, de maneira detalhada, os passos para sua aplicação, pretende-se reduzir desaproveitamentos nesses ambientes, como treinamento insuficiente, falta de supervisão, experiência, medidas de desempenho sistemáticas, responsabilidade, disponibilidade de materiais e, em geral, a ausência de uma disposição sistemática do laboratório e e dos equipamentos. Os resultados apresentam melhorias importantes em curto e médio prazo nos processos de registro e empréstimo de equipamentos e ferramentas. $\mathrm{O}$ caso analisado evidencia resultados positivos que têm o potencial de servir de exemplo para outras organizações educativas que desejarem adaptar Lean com suas ferramentas como uma alternativa viável, prática e de baixo custo.

Palavras-chave: Lean manufacturing; Lean em laboratórios; Lean no ensino superior; Kaizen; melhoria contínua.
\end{abstract}




\section{INTRODUCCIÓN}

Las organizaciones han utilizado, desde los años 50, el pensamiento Lean [1] con el objetivo de reducir o eliminar aquellas actividades que no generan valor, y simultáneamente incrementarlo en sus productos o servicios mediante un aumento de su productividad [2]. Esta necesidad surgió debido a la escasez de los recursos y la alta competencia que existía en Japón y que amenazaba la supervivencia de la Corporación Toyota Motors [3], [4].

Para lograr su objetivo este pensamiento pretende actuar sobre las causas de la variabilidad, desperdicios e inflexibilidad, que es todo lo que no se adapta a las exigencias del cliente para conseguir una mejora en calidad, costos, plazos y tiempos [5].

Lean como concepto, ha ido evolucionando y lo seguirá haciendo a través de los años [3]: ha pasado de tener un enfoque usado únicamente en plantas de producción para ser adaptado e implementado en otras industrias como la financiera, salud y en años recientes en la educación superior [6]. Esta diversificación de enfoque no es otra cosa que la adaptación de las mismas herramientas o técnicas originales a otros escenarios.

Un claro ejemplo de lo anterior es el llamado Lean Higher Education (LHE), con el que se busca que los principios y prácticas Lean usados en otras industrias, se adapten al sector de la educación superior, convirtiéndose en parte de la cultura de la institución mediante el involucramiento de las facultades y demás personal en el análisis, y mejoramiento de sus procesos a nivel administrativo y académico; todo con el fin de hacerlas más eficientes y efectivas [7].

Otro ejemplo de la adaptación de Lean en otros ambientes es Lean Laboratory (LL), que busca la mejora del desarrollo de las actividades, tanto administrativas como productivas dentro del marco de "entregar más con menos" en los laboratorios [8]. Dentro de esta aplicación específica, se presenta interés y una apreciable fuente de investigación en lo que respecta a laboratorios pertenecientes al sector de la salud [9].

A pesar de que los dos enfoques de Lean nombrados anteriormente se han planteado como áreas de investigación y aplicación independientes, existe un punto en el cual convergen, en la aplicación de herramientas Lean en los laboratorios de educación superior.

En este punto de convergencia, autores como Sreedharan y Liuo [10], RamosMartín [11], Rizvi [12] James [13] y Oberhausen y Plapper [14] están comenzando a descubrir la importancia, ventajas, desventajas, necesidades y desafíos que trae consigo esta aplicación. 
En estos laboratorios, los experimentos realizados están basados en una serie de lineamientos ya establecidos por diferentes organizaciones como ASTM (American Society for Testing And Materials), AASHTO (American Association of State Highway and Transportation Officials), Invías (Instituto Nacional de Vías), entre otras; donde los procedimientos están claramente definidos y organizados. Sin embargo, estudios experimentales son ejecutados también sobre la base de la experiencia, el ensayo y error, ya que la mayoría de ellos se realizan con el objetivo de estudiar y explorar nuevos fenómenos y horizontes.

Cabe resaltar que el desorden, mala disposición y manejo deficiente de equipos, herramientas y material son aspectos comunes para mejorar en los laboratorios académicos, generalmente causados por la falta de interés y conocimiento de los estudiantes en sus ejercicios experimentales, y por la ausencia de control por parte de la administración del laboratorio [12].

Existe poca evidencia que demuestre la introducción de Lean en estos laboratorios, donde los hábitos de trabajo y requerimientos son completamente diferentes de los laboratorios profesionales [9], [12], [15], [16]. A los estudiantes no se les paga por el trabajo que realizan en el laboratorio, es más bien una diversión para ellos el estar allí y jugar con los instrumentos y materiales. Ellos no toman o están dispuestos a asumir la responsabilidad del trabajo de laboratorio, sino que es parte de su curso, además de ser una actividad adicional para ellos [12]. Es por lo anterior que para la buena ejecución y éxito de las actividades realizadas en un laboratorio, la dirección y gestión de las instalaciones y la disposición de maquinaria, herramientas y material en el lugar y momento adecuado juegan un papel fundamental. Aspectos en los cuales estas instalaciones académicas tienden a fallar frecuentemente.

Es por esto que la generación de evidencias para las mejoras que se pueden proveer en los laboratorios de educación superior, gracias a la gran variedad de herramientas Lean existentes, permitirían abrir una puerta de mejora continua en un sector importante del servicio de la educación, como es la formación basada en la experiencia e investigación.

El objetivo del presente artículo es proponer una herramienta guía para la implementación de Lean en laboratorios de educación superior en Colombia, y su evaluación en términos del grado de mejoras que se puedan alcanzar en este entorno, teniendo como caso de aplicación el laboratorio de suelos y pavimentos de la Escuela Colombiana de Ingeniería Julio Garavito.

Para el logro de este objetivo, el artículo está estructurado de la siguiente manera: revisión de la literatura de la aplicación de Lean en laboratorios académicos para identificar allí hábitos de trabajo, requerimientos y aspectos comunes a mejorar. Continúa con una descripción detallada de la herramienta guía. Posteriormente, se 
muestran los inconvenientes identificados en el laboratorio y cómo se abordaron desde la herramienta guía, para finalmente exponer las conclusiones del estudio.

\section{REVISIÓN DE LA LITERATURA}

A pesar del éxito bien documentado de Lean manufacturing en las últimas décadas, en general los laboratorios han sido lentos en adoptar sus principios para mejorar su desempeño y los servicios que proporcionan a sus clientes. No obstante, esta es una transformación que no puede evitarse: debido a ser un ambiente rico en datos y familiarizado con los principios de medición, los laboratorios están bien posicionados para implementar y aprovechar efectivamente estas técnicas de mejoramiento [17].

\subsection{Lean en laboratorios}

La literatura sobre la aplicación de Lean en laboratorios presentada en la tabla 1, donde se muestra que existe un gran interés en la evaluación del impacto de Lean en laboratorios relacionados con el sector de la salud, hallazgos que pueden llegar a ser adaptados e implementados en laboratorios de otra índole.

En laboratorios del sector de la salud, Halwach-Baumann [18] sostiene que los ensayos son una parte fundamental en la toma de decisiones, y a pesar de presentar grandes desafíos, como la preferencia por los métodos tradicionales, un flujo de trabajo complejo y una variabilidad marcada en el número de muestras [19], los principios básicos del pensamiento Lean pueden ser aplicados, con el fin de maximizar la productividad, reducir costos y asegurar resultados de calidad [19], [20]. Una hipótesis que aplicada a laboratorios de educación superior sigue siendo válida.

El proceso necesario para crear un ambiente Lean en el laboratorio varía considerablemente en función de los tipos de ensayos que se realizan en cada instalación y el número de muestras que allí llegan. El procedimiento básico de aplicación de Lean se mantiene e incluye la observación y documentación del ambiente y flujo de trabajo actual, la identificación de desperdicios en los materiales y actividades, el rediseño de procesos para garantizar el flujo continuo del trabajo, y finalmente una evaluación de mejora continua [19].

Existen diferentes maneras de comenzar a implementar Lean en un laboratorio, por ejemplo, se pueden identificar actividades que no generen valor e intentar eliminarlas, esto mediante la reducción mínima de personal por ensayo; otro ejemplo es implementar cambios en el proceso que mejoren el flujo de trabajo, en algunos casos es posible lograr esto mediante la eliminación de la necesidad de procesar muestras en lote, el dejar muestras en espera y que lleguen más de las mismas para completar la capacidad del equipo a utilizar disminuye la velocidad del proceso en general [20]. 


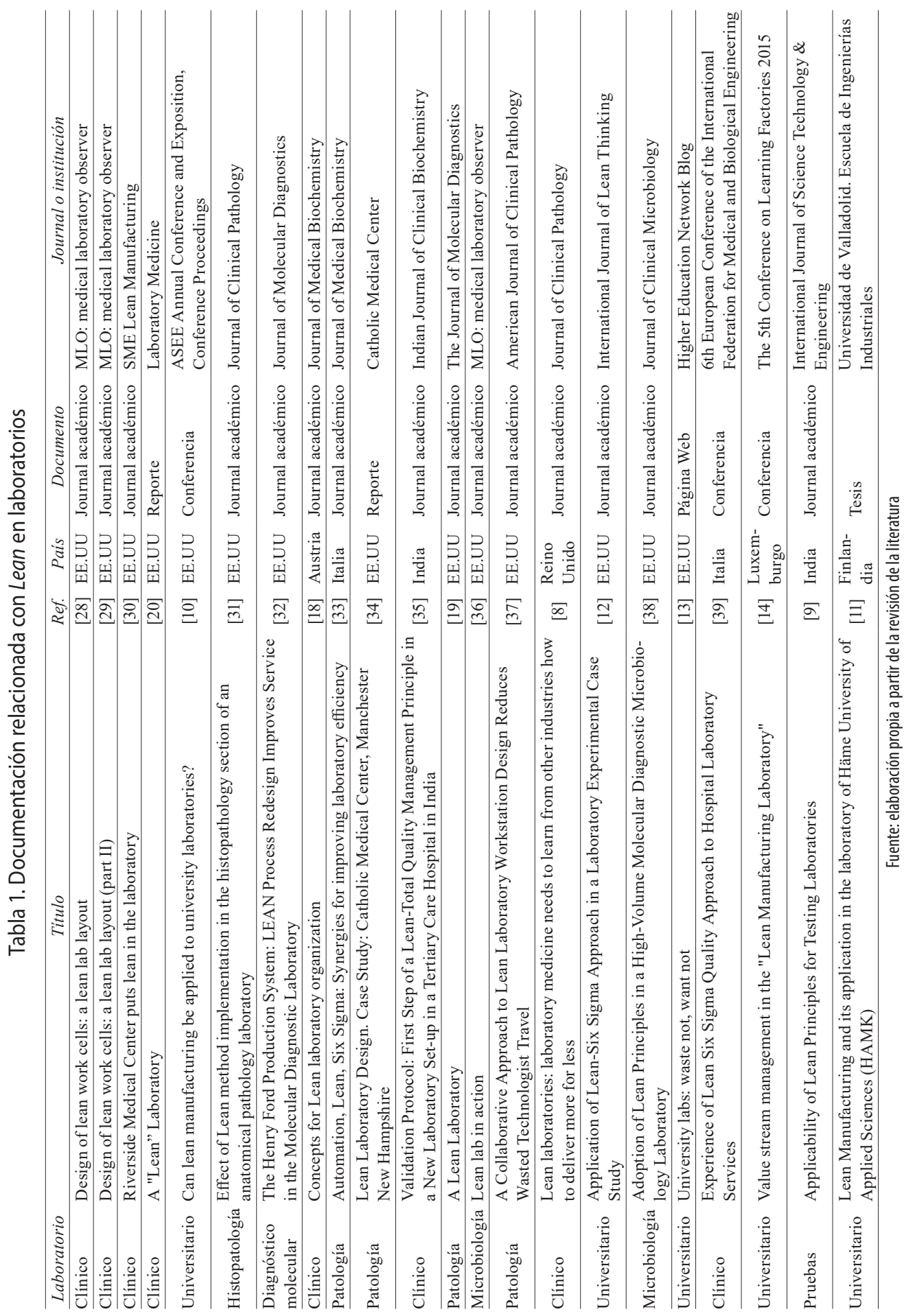


Estas son solo algunas de las estrategias que se pueden generar en el proceso de implementación de Lean en laboratorios; existe evidencia de otras más de esta índole en diferentes centros médicos y académicos de Europa, Asia y América tal como se observa en la tabla 1.

De manera general, se observa que la falta de comunicación, la necesidad de procesar ensayos en lotes, el no querer estandarizar procesos y la falta de orden en la planta y en el almacenamiento, son problemas comunes que se lograron solucionar con diferentes herramientas Lean como Kaizen o Kanban, complementado con rediseños de planta y una adecuada capacitación de los trabajadores en estos temas.

\subsection{Lean en laboratorios académicos}

Los experimentos en el laboratorio son una actividad convencional realizada por instituciones académicas, el gobierno y organizaciones privadas. Estos estudios experimentales proveen bases para nuevos inventos en el campo de la ciencia y la ingeniería [12].

Como se puede comprobar en la figura 1 , se destaca que el $77 \%$ de los artículos encontrados en la revisión literaria tratan sobre la aplicación de Lean en laboratorios del sector salud, mientras que su aplicación en laboratorios universitarios y de pruebas representa únicamente el $18 \%$ y $5 \%$ respectivamente.

A pesar de esta limitada información encontrada en laboratorios académicos, se puede apreciar que los desperdicios son un producto común. Entrenamiento insuficiente, falta de supervisión, experiencia, medidas de rendimiento sistemáticas, responsabilidad, disponibilidad de materiales y en general, ausencia de una disposición sistemática del laboratorio y del equipo, son algunas de las razones comunes que generan despilfarros. A pesar que no todas estas causas se tienen en cuenta, algunas áreas pueden mejorar considerablemente mediante la eliminación del desperdicio producido en el estudio experimental en laboratorio [12].

En cuanto a los casos de estudio, la literatura sugiere que existe una variedad de herramientas Lean útiles y completamente aplicables en laboratorios universitarios. No obstante, cada una de ellas debe tener un objetivo claro y un control de los resultados al aplicarlas. Algunas de ellas son el Value Stream Mapping (VSM), 5's, gestión visual, Heijunka y Total Productive Maintenance (TPM); todas aplicadas bajo un marco de mejora continua.

Este marco de mejora continua demuestra que el éxito de Lean en laboratorios académicos se fundamenta en un análisis de la situación actual con el objetivo de identificar todas las clases de desperdicios posibles, una identificación de las causas 


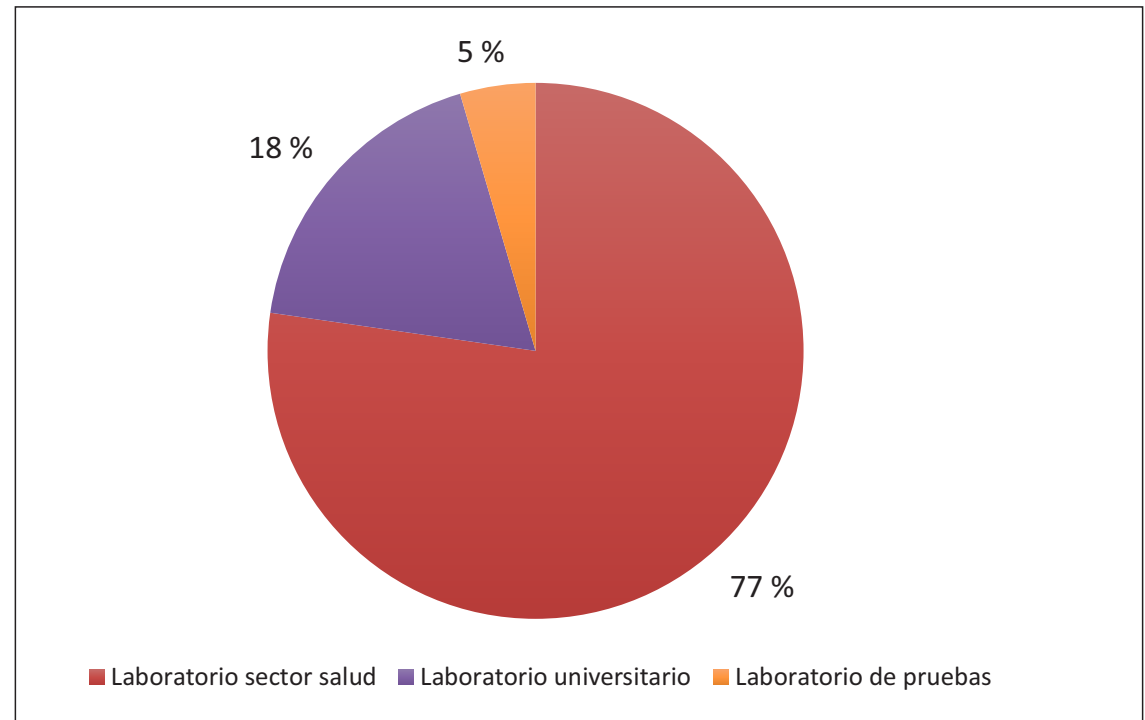

Figura 1. Tipos de laboratorios con evidencia de implementación Lean Fuente: elaboración propia a partir de la revisión de la literatura

raíces de estos desperdicios, el establecimiento de unos objetivos o metas, un plan de acción para reducirlos o eliminarlos, y finalmente, una evaluación de este plan de acción con el fin de establecer lo logrado y las futuras mejoras.

No obstante, aun cuando algunos de los casos encontrados en la literatura evidencian mejoras en los procesos de laboratorios, no queda claro si existió una estrategia de estandarización para mantener los cambios propuestos, lo cual no permite determinar el verdadero éxito de la aplicación de diferentes herramientas Lean en estos ambientes.

También es importante resaltar que tal como lo sugiere Ramos [11], los cambios en la forma de trabajo de los laboratorios no se puede dar de forma abrupta, ya que los trabajadores no estarán preparados para cambios a gran escala y los resultados esperados se verán comprometidos. El proceso de implementación de Lean en cualquier ambiente, debe generarse de forma gradual con el fin de asegurar su éxito, los cambios paulatinos a largo plazo darán como resultados grandes cambios.

\subsection{Iniciativas de implementación Lean}

A pesar de ser una de las metodologías más poderosas para la mejora continua, cerca de dos terceras partes de los intentos de implementación de Lean terminan en fracasos, y menos de una quinta parte mantienen sus resultados a largo plazo [21], es por esto que el proceso de implementación es crucial para el éxito de este ejercicio. 
Existen varias iniciativas de implementación de Lean en la literatura, las cuales se pueden agrupar en cinco categorías: herramientas guía conceptuales, herramientas guía de implementación, listas de verificación descriptivas y listas de verificación de evaluación, incluso algunos investigadores nombran sus procesos de implementación como un estilo descriptivo [22]. Aquellas implementaciones más exitosas han sido las presentadas como mapas de ruta o herramientas guía. Pese a ello, se ha reportado una baja utilización de iniciativas Lean y una baja tasa de éxito, lo cual parece ser atribuido a que muchas de estas iniciativas no son consideradas comprensibles por parte de quienes las utilizan [23].

Aquellas implementaciones más exitosas han sido las presentadas como mapas de ruta o herramientas guía. Pese a ello, se ha reportado una baja utilización de iniciativas Lean y una baja tasa de éxito, lo cual parece ser atribuido a que muchas de estas iniciativas no son consideradas comprensibles por parte de quienes las utilizan [23]. Dado lo anterior, se procederá a desarrollar una herramienta guía que permita la implementación de Lean en laboratorios de educación superior.

\section{HERRAMIENTA GUÍA}

Una herramienta guía se describe como unos lineamientos a seguir de manera sistemática y exhaustiva por parte de una organización. Esta herramienta debe contar con unos criterios fáciles de entender, simples y claros, capaces de responder preguntas como ¿Cómo se hace? y ¿Qué es? [24]. A partir de la revisión de la literatura presentada anteriormente y las investigaciones realizadas por [24] y [22], es posible observar una falencia en la secuencia, control, monitoreo y registro de las herramientas propuestas, razón por la cual su aplicabilidad y reproducción en otros ambientes o áreas no ha sido posible.

En la figura 2 se presenta la herramienta guía propuesta, la cual comprende quince pasos, divididos en cuatro fases para la implementación de Lean en laboratorios de educación superior (LES), esta incluye aquellos elementos ausentes identificados en la literatura y supone una solución a los problemas típicos, mencionados con anterioridad en los laboratorios de esta índole. Se considera apropiado la utilización de Kaizen para su ejecución, ya que esta herramienta aplica el ciclo PDCA (Plan-Do-Check-Act) como base de su funcionamiento [25].

El Kaizen es una herramienta que permite una mejora continua en todo el flujo de valor o en procesos individuales a los cuales se les quiera agregar más valor con menos desperdicios [25]. Esta herramienta se adapta a toda clase de proyectos, desde aquellos que únicamente requieren una semana para implementarse, hasta aquellos que 
requieren más de tres meses. Dependiendo de la envergadura del proyecto, el Kaizen usado será diferente: Quick, Standard, Major o Advanced Kaizen.

\subsection{Fase de estructuración}

Esta es la fase de inicio, en ella se busca determinar los problemas, sus causas raíces y los objetivos de mejora o KPI (Key Performance Indicators). Esta determinación la debe realizar un grupo de trabajo asignado con conocimientos en temas Lean y los procesos ejecutados en el laboratorio.

Es importante en esta etapa establecer unos canales de comunicación entre el grupo de trabajo y todos los usuarios y trabajadores del laboratorio, ya que esta será la fuente principal de información para las siguientes etapas.

\subsection{Fase de ejecución}

En esta etapa se tiene por meta establecer e implementar los cambios que se consideren necesarios para cumplir con los KPI. Para el cumplimiento de estos objetivos, es necesario hacer un análisis exhaustivo de las posibles herramientas Lean a utilizar.

Gracias al gran número de herramientas existentes, Lean tiene la capacidad de adaptarse a diferentes ambientes; dentro de las investigaciones adelantadas en el área de los laboratorios se puede observar que herramientas como VSM (value stream mapping), 5's, gestión visual, Heijunka y TPM han generado resultados positivos. No obstante, tal como sostienen Ramos [11] y Rizvi [12], existen muchas más herramientas que pueden ayudar a mejorar los laboratorios, y que deberían ser exploradas con el objetivo de tener un espectro menos limitado sobre la influencia de Lean en este tipo de instalaciones.

Para la selección de estas herramientas se puede hacer uso de la tabla 2, la cual es una compilación de diferentes KPI típicos de LES identificados en la literatura y las diferentes herramientas con los cuales fueron logrados. Esta información fue obtenida luego del análisis de los veintidós autores mostrados en la revisión de la literatura.

A manera de ejemplo, se puede decir que hay evidencia de que cuando se quiera reducir el tiempo de búsqueda de herramientas dentro del laboratorio, el uso de 5's, gestión visual, 5 whys y Kaizen es una solución adecuada.

Además de analizar la pertinencia de herramientas Lean, es importante generar una gran variedad de soluciones para depurar y seleccionar aquellas con mayor potencial de éxito, por su reducción de costos, mejora de la calidad, viabilidad técnica de las acciones de mejora y por su contribución a un posicionamiento para que la compañía logre un mayor crecimiento [26]. 


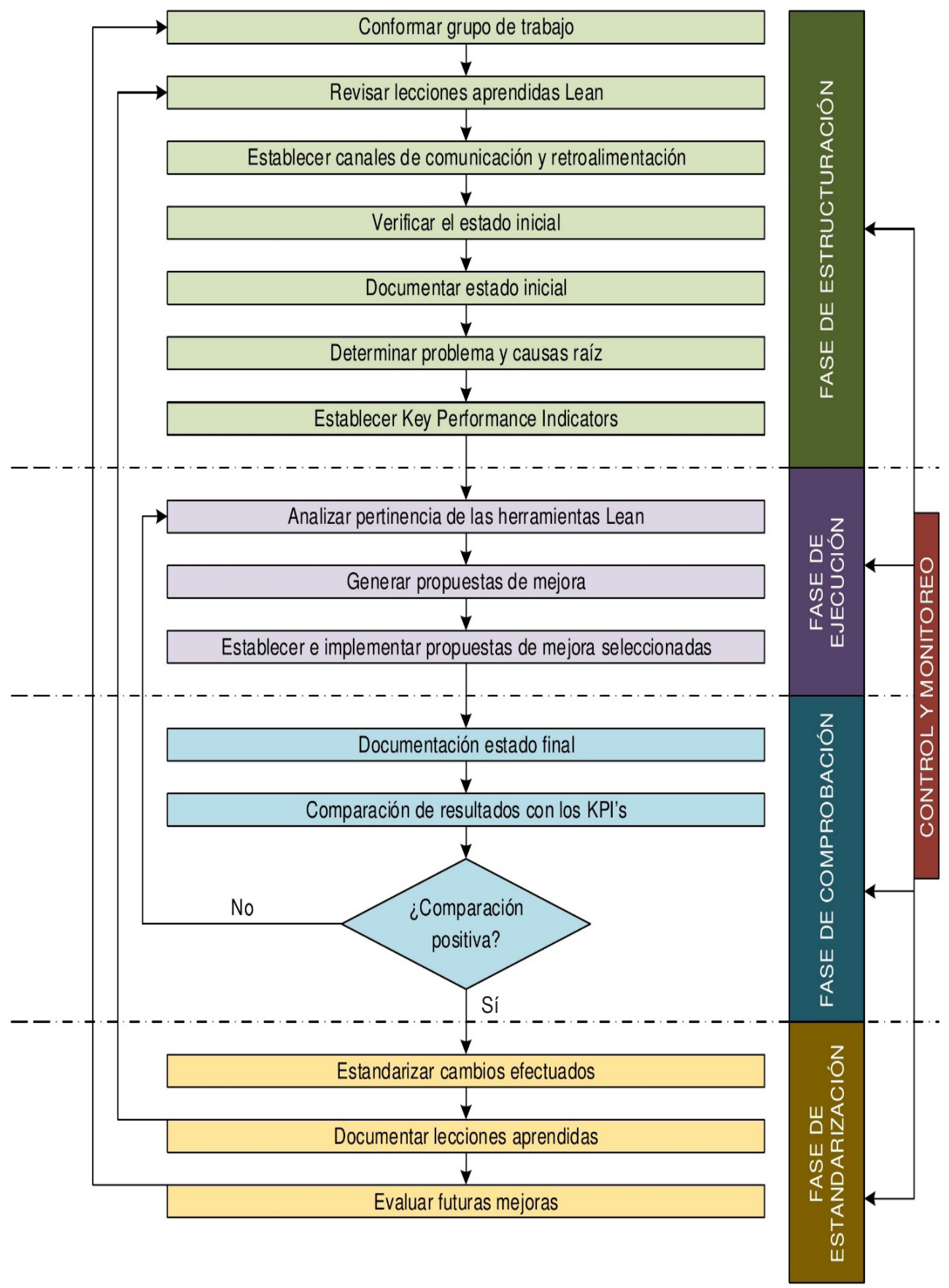

Figura 2. Herramienta guía propuesta para implementación de Lean en LES Fuente: elaboración propia 
Tabla 2. Pertinencia herramienta Lean ante diferentes KPI

\begin{tabular}{|c|c|c|c|c|c|c|c|c|}
\hline KPI clásicos de laboratorio & $i^{n}$ & 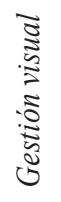 & 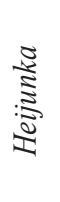 & 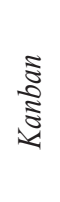 & $\underset{n}{\frac{2}{3}}$ & 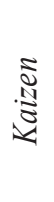 & 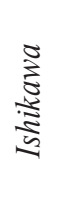 & $\underset{\Delta}{\Xi}$ \\
\hline Reducción en tiempo de búsqueda de herramientas & $\mathrm{X}$ & $\mathrm{X}$ & & & $\mathrm{X}$ & $\mathrm{X}$ & & \\
\hline Reducción de diligenciamiento de formatos & & $\mathrm{X}$ & & & $\mathrm{X}$ & $\mathrm{X}$ & & \\
\hline Reducción en tiempos de ensayos & & & $\mathrm{X}$ & $\mathrm{X}$ & $\mathrm{X}$ & $\mathrm{X}$ & $\mathrm{X}$ & $\mathrm{X}$ \\
\hline $\begin{array}{l}\text { Reducción en tiempo de búsqueda de información de } \\
\text { maquinaria }\end{array}$ & $\mathrm{X}$ & $\mathrm{X}$ & & & $\mathrm{X}$ & $\mathrm{X}$ & & \\
\hline $\begin{array}{l}\text { Reducción de tiempo del proceso de préstamo de equipos y } \\
\text { herramientas }\end{array}$ & & & & $\mathrm{X}$ & $\mathrm{X}$ & $\mathrm{X}$ & $\mathrm{X}$ & \\
\hline $\begin{array}{l}\text { Reconocimiento de la disposición del espacio para la } \\
\text { reducción de transporte }\end{array}$ & $\mathrm{X}$ & $\mathrm{X}$ & & & $\mathrm{X}$ & $\mathrm{X}$ & & $\mathrm{X}$ \\
\hline $\begin{array}{l}\text { Actualización permanente de sistema inventario del } \\
\text { laboratorio }\end{array}$ & & $\mathrm{X}$ & & $\mathrm{X}$ & & & & \\
\hline $\begin{array}{l}\text { Aumento de la flexibilidad de los sistemas de inventario, } \\
\text { registro y préstamo }\end{array}$ & & $\mathrm{X}$ & & $\mathrm{X}$ & & & & \\
\hline $\begin{array}{l}\text { Aumento de la disponibilidad de herramientas y materiales } \\
\text { en el laboratorio }\end{array}$ & $\mathrm{X}$ & $\mathrm{X}$ & & $\mathrm{X}$ & & $\mathrm{X}$ & & \\
\hline $\begin{array}{l}\text { Reducción de mantenimientos correctivos en los equipos } \\
\text { (aumento de mantenimientos preventivos) }\end{array}$ & & $\mathrm{X}$ & $\mathrm{X}$ & $\mathrm{X}$ & & $X$ & & \\
\hline
\end{tabular}

Fuente: elaboración propia a partir de la revisión de la literatura

\subsection{Fase de comprobación}

Una vez implementadas las mejoras, se observan los procesos durante un periodo de tiempo pertinente, el cual dependerá de la complejidad del proceso modificado, con el objetivo de evaluar los cambios en términos de los KPI establecidos. Este ejercicio permite identificar los cambios de forma cualitativa y cuantitativa, y a partir de estos resultados se determina qué tan efectivas son las soluciones propuestas.

\subsection{Fase de estandarización}

Aquellas mejoras que generan un impacto positivo en el rendimiento de los procesos, son estandarizadas con el objetivo de mantenerlas y no desmejorar en ningún momento. Esta estandarización puede ir desde la elaboración de un manual simple, hasta la automatización de procesos. 
Cabe aclarar que este proceso de mejora se debe realizar con ayuda de los trabajadores del lugar, ya que de esta manera se espera que ellos se sientan parte del proceso y tengan la libertad de mejorar los procesos trabajados u otros procesos del laboratorio no contemplados anteriormente.

Una vez presentada la herramienta guía, se evaluará su eficiencia con ayuda de un caso de aplicación.

\section{CASO DE APLICACIÓN}

Para evaluar la herramienta guía propuesta en la gestión de los laboratorios académicos, se tomará como caso de aplicación el laboratorio de suelos y pavimentos de la Escuela Colombiana de Ingeniería (ECI), ya que este presenta hábitos de trabajo, requerimientos y aspectos a mejorar típicos encontrados en la literatura para estos ambientes.

Se evidenció en el momento del diagnóstico que durante la logística y el proceso de traslado, existía una ausencia de prácticas adecuadas para la disminución de los desperdicios comunes que la literatura presenta, y los resume cómo "la ausencia de una disposición sistemática del laboratorio y del equipo" [12], la falta de orden y de bases de datos con registros de información y movimientos de los equipos y herramientas, amenazan el buen funcionamiento del lugar, generan dificultades para la adaptación de nuevas personas a este ambiente de trabajo y dan como resultado pérdidas de tiempo, esfuerzo y dinero.

Al ser Lean un proceso de mejora que debe ser tomado de manera paulatina, la mejora de todos los inconvenientes y desperdicios identificados no entraron durante la aplicación de Lean en el laboratorio, sin embargo, se considera un buen inicio para generar allí grandes cambios a futuro.

Para este caso en particular se utilizó un Standard Kaizen, del cual se esperaba que su implementación durara máximo un mes, plazo estipulado por el director del laboratorio. El formato del Kaizen diligenciado para este proceso de mejora se presenta en la figura 3, y un despiece de este se muestra en la figura 4.

\subsection{Fase de estructuración}

Se seleccionaron los procesos de registro y préstamo de equipo, y proceso de préstamo de herramientas para mejorar. La información se recolectó a través de una observación directa del lugar y los procesos por un periodo de dos semanas. Una vez se obtuvo un punto de vista propio, se comenzó a complementar con la opinión de los trabajadores del laboratorio (secretaria, laboratoristas y docentes) recolectada mediante entrevistas individuales; esto ofreció un espectro amplio de todo lo que sucedía alrededor de los 
procesos estudiados, tal como el estado actual, sus falencias e incluso mejoras que los mismos entrevistados propusieron cuando lo consideraron relevante.

A partir de esta observación directa y de las entrevistas, se generó un análisis de los problemas encontrados para la determinación de sus causas raíz, esto con la aplicación de las herramientas 5 why's e Ishikawa.

Con la información recolectada de los procesos de estudio, fue posible establecer que el registro de maquinaria, mediante sus hojas de vida, era altamente manual y con varios reprocesos. Adicionalmente, se observó la inflexibilidad del sistema de préstamo y la desactualización del inventario.

En el proceso de registro de las hojas de vida se usaba un formato extenso (tres hojas) difícil de diligenciar y completamente manual, este ejercicio se realizaba en el lugar donde la máquina estuviera ubicada en las instalaciones, para posteriormente colgarlo en un tablero a la espera de que la secretaria lo digitalizara, imprimiera y almacenara en un estante físico alejado de su puesto de trabajo, proceso que en total tomaba cerca de dos horas si se ejecutaba de manera continua.

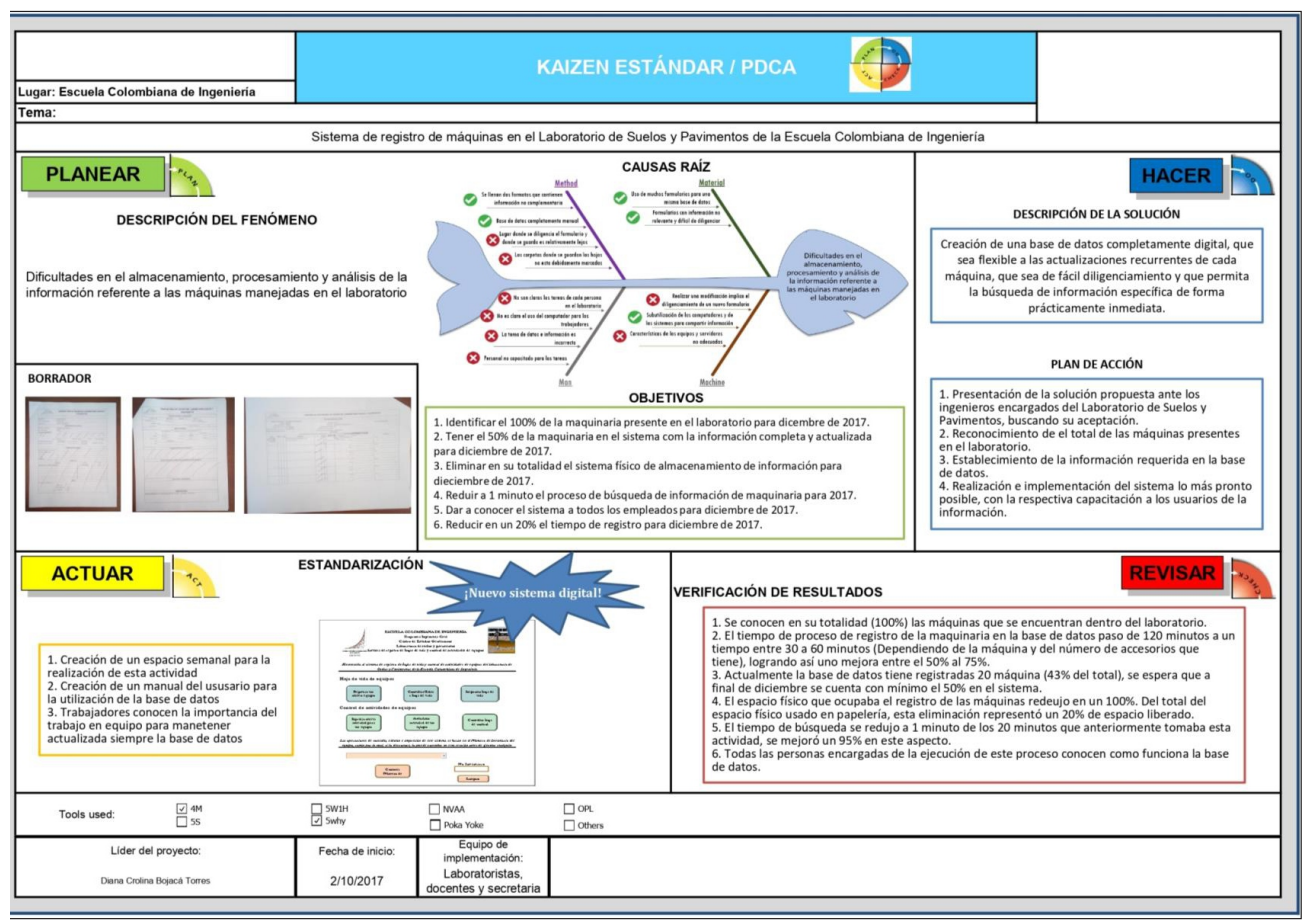

Figura 3. Formato Kaizen estándar diligenciado

Fuente: elaboración propia 


\section{PLANEAR}

DESCRIPCIÓN DEL FENÓMENO

Dificultades en el almacenamiento, procesamiento y análisis de la información referente a las máquinas manejadas en el laboratorio

\section{BORRADOR}

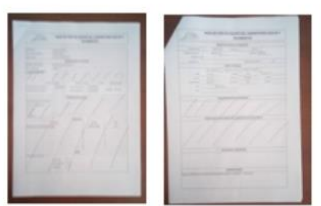

\section{CAUSAS RAIZ}

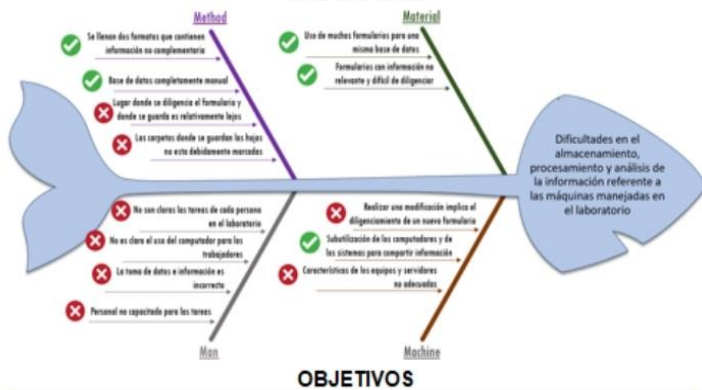

OBJETIVOS

1. Identificar el $100 \%$ de la maquinaria presente en el laboratorio para dicembre de 2017 2. Tener el $50 \%$ de la maquinaria en el sistema com la información completa y actualizada para diciembre de 2017.

3. Eliminar en su totalidad el sistema físico de almacenamiento de información para dieciembre de 2017.

4. Reduir a 1 minuto el proceso de búsqueda de información de maquinaria para 2017.

5. Dar a conocer el sistema a todos los empleados para diciembre de 2017.

6. Reducir en un $20 \%$ el tiempo de registro para diciembre de 2017.

\section{VERIFICACIÓN DE RESULTADOS}

1. Se conocen en su totalidad (100\%) las máquinas que se encuentran dentro del laboratorio. 2 . El tiempo de proceso de registro de la maquinaria en la base de datos paso de 120 minutos a un tiempo entre 30 a 60 minutos (Dependiendo de la máquina y del número de minutos a un tiempo entre 30 a 60 minutos (Dependiendo de la máquir

accesorios que tiene), logrando asi uno mejora entre el $50 \%$ al $75 \%$. 3. Actualmente la base de datos tiene registradas 20 máquina ( $43 \%$ del total), se espera que a final de diciembre se cuenta con mínimo el $50 \%$ en el sistema.

4. El espacio físico que ocupaba el registro de las máquinas redeujo en un $100 \%$. Del total del espacio físico usado en papelería, esta eliminación representó un $20 \%$ de espacio liberado. 5 . El tiempo de búsqueda se redujo a 1 minuto de los 20 minutos que anteriormente tomaba esta actividad, se mejoró un $95 \%$ en este aspecto.

6. Todas las personas encargadas de la ejecución de este proceso conocen como funciona la base de datos.

\section{HACER}

DESCRIPCIÓN DE LA SOLUCIÓN

Creación de una base de datos completamente digital, que sea flexible a las actualizaciones recurrentes de cada máquina, que sea de fácil diligenciamiento y que permita la búsqueda de información específica de forma prácticamente inmediata.

\section{PLAN DE ACCIÓN}

1. Presentación de la solución propuesta ante los ingenieros encargados del Laboratorio de Suelos $y$ Pavimentos, buscando su aceptación

2. Reconocimiento de el total de las máquinas presentes en el laboratorio.

3. Establecimiento de la información requerida en la base de datos.

4. Realización e implementación del sistema lo más pronto posible, con la respectiva capacitación a los usuarios de la información.

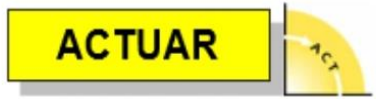

1. Creación de un espacio semanal para la realización de esta actividad 2. Creación de un manual del ususario para la utilización de la base de datos 3. Trabajadores conocen la importancia del trabajo en equipo para manetener actualizada siempre la base de datos

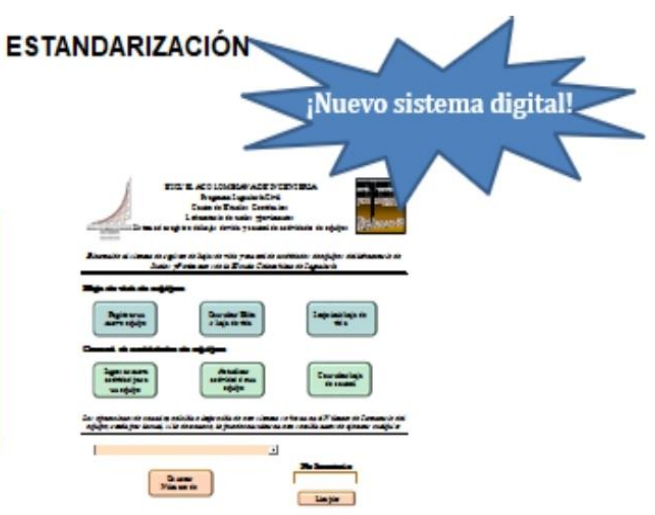

Figura 4. Despiece formato Kaizen estándar diligenciado

Fuente: elaboración propia 
En dicho estante los formatos se almacenaban en unas carpetas que en su exterior no estaban marcadas adecuadamente, por lo cual la búsqueda de cualquier formato implicaba abrir todas las carpetas para conocer el contenido de cada una, tomando aproximadamente de veinte minutos (figura 5).

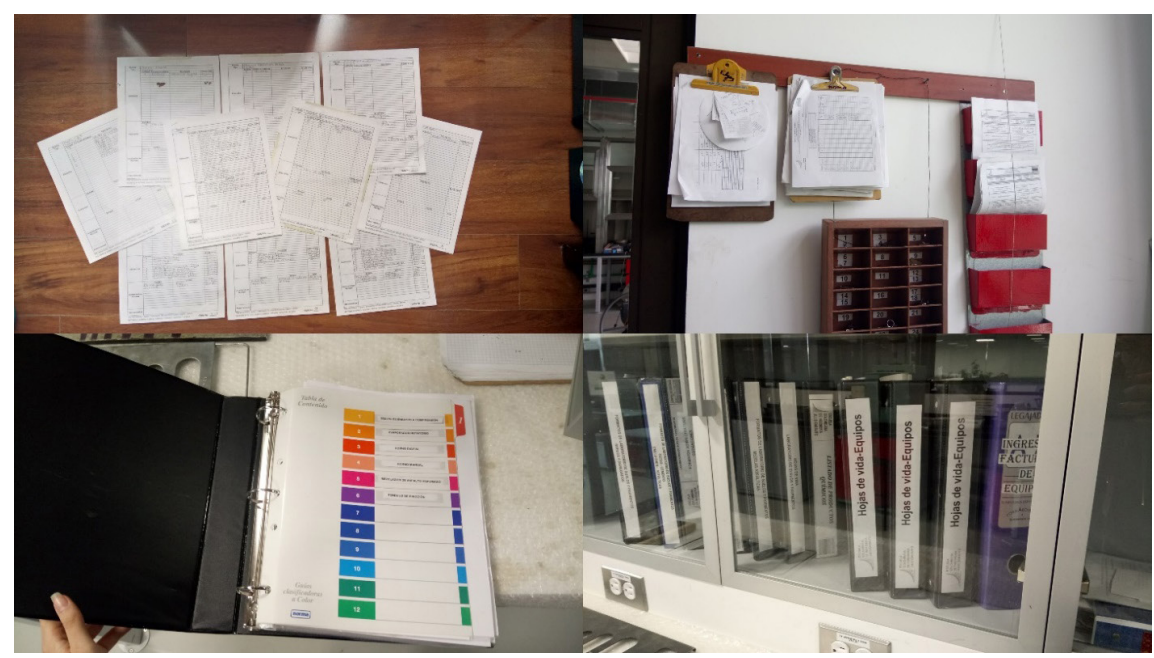

Figura 5. Manejo de la información de registro Fuente: elaboración propia

Por su parte, el sistema usado para mantener el control de préstamo de herramientas se encontraba desactualizado en cuanto a inventario, y además era poco flexible en la edición de registros de préstamos ya existentes, por lo que una solicitud podía tomar hasta veinte minutos. Esta inflexibilidad y desactualización dio como resultado que el sistema entrara en desuso.

La determinación de las causas raíz por medio del diagrama Ishikawa y 5 whys mostró que la complejidad e inflexibilidad de los procesos, sumado al poco uso de tecnología eran los principales detonantes de la poca eficiencia de dichos procesos.

A partir de los resultados encontrados, y usando como base los KPI clásicos de los laboratorios mostrados en la tabla 2, se establecieron las metas para los KPI para este proyecto, los cuales fueron:

- Identificar el $100 \%$ de la maquinaria y herramienta presente en el laboratorio para diciembre de 2017.

- Tener el $50 \%$ de la maquinaria en el sistema con la información completa y actualizada para diciembre de 2017. 
- Eliminar en su totalidad el sistema físico de almacenamiento de información para diciembre de 2017.

- Reducir a un minuto el proceso de búsqueda de información de maquinaria para diciembre de 2017.

- $\quad$ Reducir en un $20 \%$ el tiempo de registro para diciembre de 2017.

- Implementar nuevamente el uso del sistema de préstamo y reducir en un $50 \%$ el tiempo de proceso para diciembre de 2017.

\subsection{Fase de ejecución}

Para este caso en particular se tomó un periodo de dos semanas para establecer propuestas de mejora y seleccionar las más adecuadas. Una vez seleccionadas, se presentaron ante los encargados de laboratorio para su aceptación, y posteriormente se generó una metodología de implementación y capacitación con el objetivo de que los trabajadores del laboratorio se apropiaran de dicha solución y la implementaran, trabajo que tomó otras dos semanas.

A la luz de las causas raíz identificadas y los objetivos establecidos, se decidió que la propuesta de mejora más viable era la creación de una base de datos completamente digital, flexible a las actualizaciones recurrentes de cada máquina, que fuera de fácil diligenciamiento y permitiera la búsqueda de información específica de forma prácticamente inmediata en el caso del proceso de registro; en el caso del proceso de préstamo, una aplicación digital y flexible a cambios en las solicitudes de préstamo y en el inventario. Estas dos propuestas permitirán reducir desperdicios en tiempo, espacio y dinero al ser implementadas.

Las figuras 6 y 7 muestran la interfaz de estos dos sistemas, cabe señalar que uno de los objetivos primordiales es que fuera de fácil uso y acceso para el personal del laboratorio, motivo por el cual se desarrollaron en hojas de cálculo de Microsoft Excel.

\subsection{Fase de comprobación}

Al momento de evaluar el impacto de las propuestas de mejora, los resultados fueron positivos. En cuanto al tiempo del proceso de registro, la eliminación del diligenciamiento manual y demás reprocesos permitió una reducción mayor a la planteada en los objetivos (figura 8a), de cerca del $50 \%$.

Con respecto a los tiempos de búsqueda en la base de datos de registro y los tiempos de préstamo, los nuevos sistemas lograron cumplir con sus objetivos (figura 8 b y c), en el primer caso se redujo un $95 \%$ el tiempo y en el segundo un $50 \%$. 


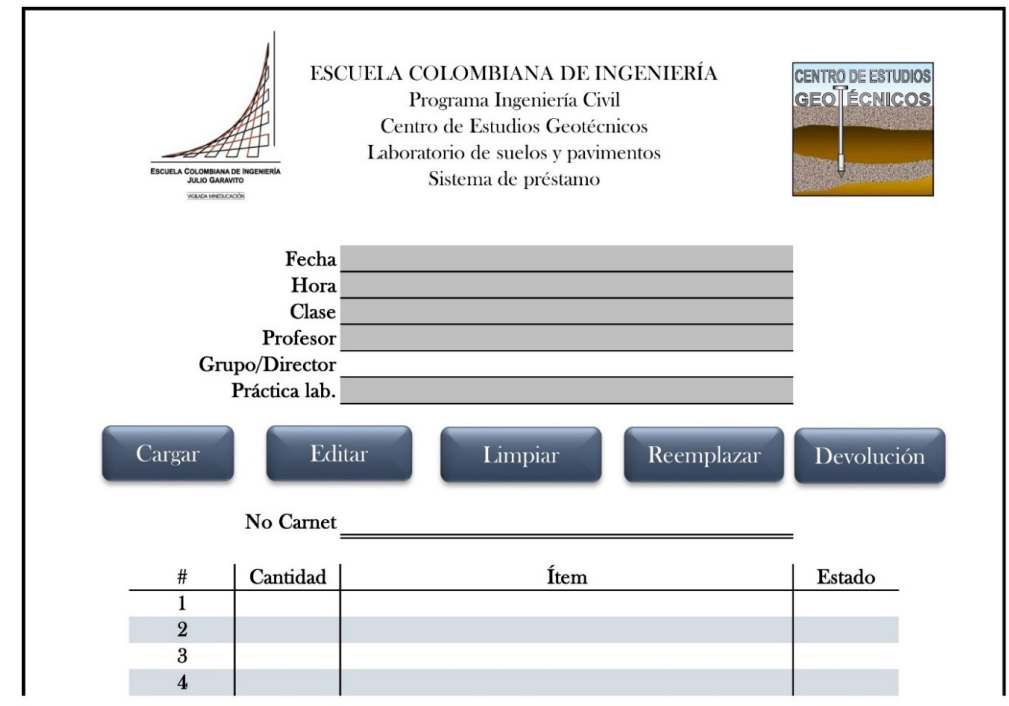

Figura 6. interfaz sistema de préstamo

Fuente: elaboración propia

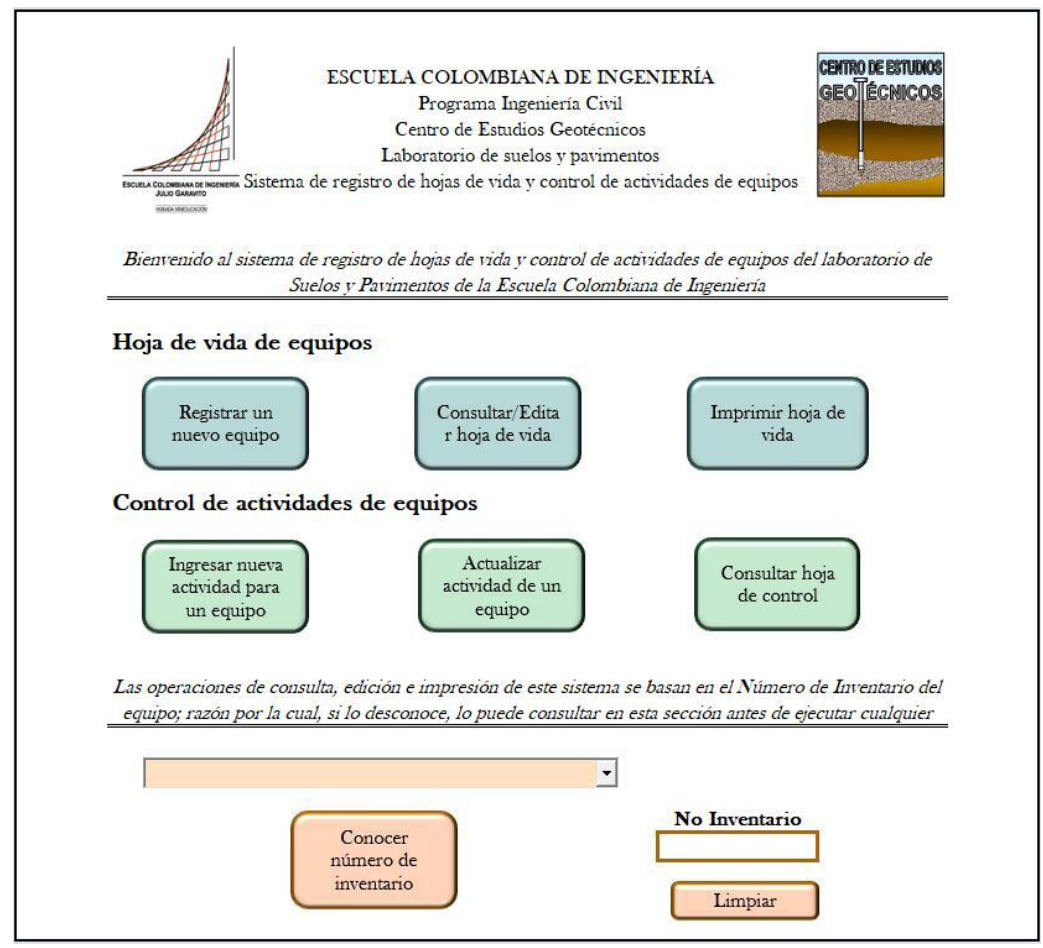

Figura 7. Interfaz sistema de registro

Fuente: elaboración propia 
Por otra parte, en cuanto al registro de las máquinas en la nueva base de datos, aunque se lograron identificar todas (47 en total), para el momento de la evaluación no se cumplió con el objetivo del $50 \%$ establecido en la fase de estructuración del proyecto, ya que se registraron 20 equipos que equivalen al $43 \%$. No obstante, se considera un logro, puesto que no se encuentra muy alejado de lo esperado. De acuerdo con la teoría de las metas, los empleados están más motivados para alcanzar metas específicas cuando estas tienen en el carácter de ser retadoras [27] (figura 8d).

Finalmente, en cuanto a espacio físico utilizado, también fue posible evidenciar que este se redujo considerablemente (figura 9) ya que no es necesario imprimir y almacenar como sucedía antes. Este cambio trae consigo, aunque no se cuantificó en este proyecto, una reducción en uso de recursos como papel, energía, tinta y elementos de papelería como carpetas.
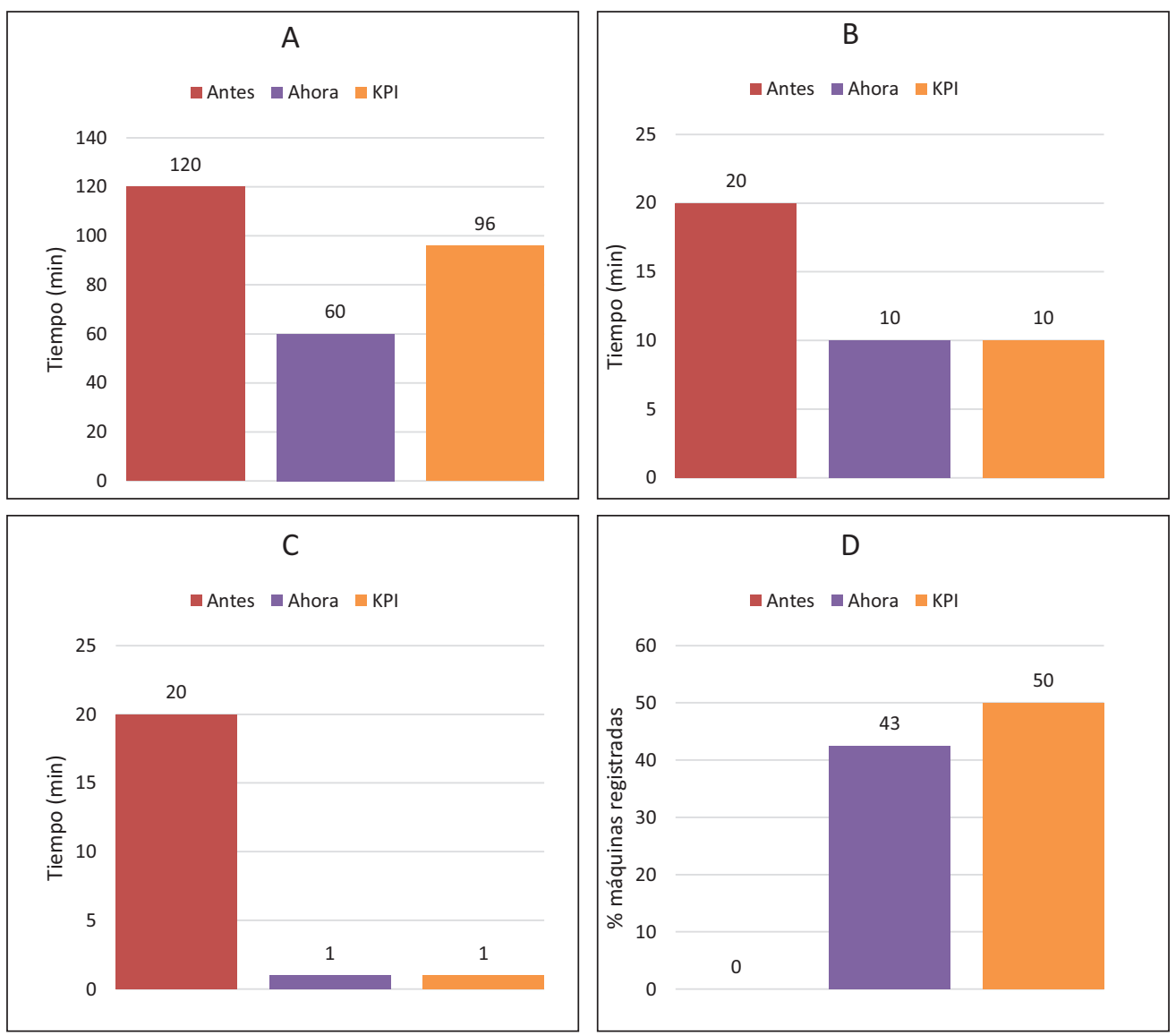

Figura 8. Resultados de: a) tiempo de registro, b) tiempo de solicitud de préstamo, c) tiempo de búsqueda y d) máquinas registradas en el sistema

Fuente: elaboración propia 


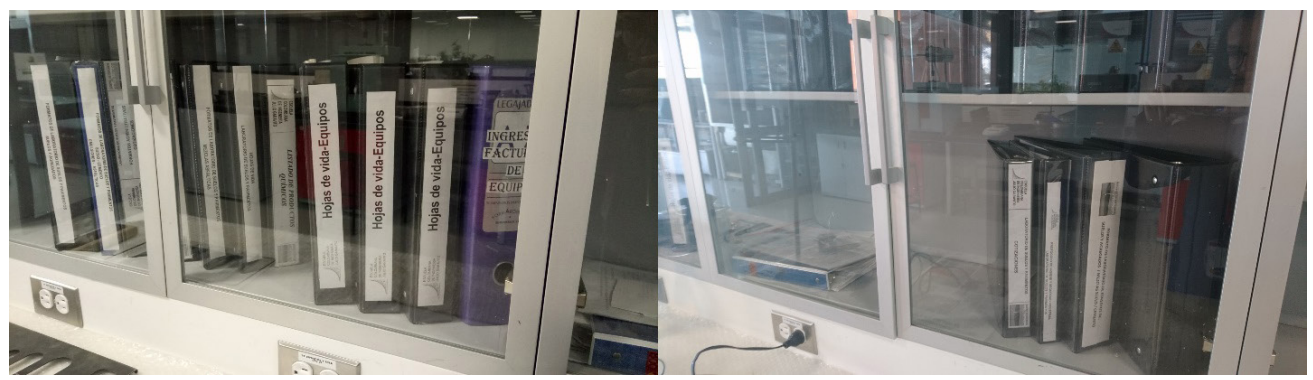

Figura 9. Liberación de espacio en sistema de almacenamiento de registro Fuente: elaboración propia

\subsection{Fase de estandarización}

Debido al impacto positivo presentado por estas propuestas de implementación, se tomó la decisión de estandarizarlas dentro del laboratorio. Para este caso en particular se elaboró un manual del usuario para el uso de ambos sistemas.

Esto permitirá que ante un cambio de personal las lecciones aprendidas y el uso de las herramientas puedan ser utilizadas al quedar documentadas.

\section{CONCLUSIONES}

Este documento presenta una herramienta guía que sirve para la implementación de Lean en ambientes de laboratorios de educación superior. Tal como se pudo observar en el caso de aplicación del laboratorio, Lean es un enfoque que cuenta con la flexibilidad para ser adaptado e implementado en ambientes diferentes a la manufactura.

También es importante reconocer que el éxito de su uso dependerá del plan de acción utilizado para implementarlo. Entre más simple y comprensible sea, entre más personas del lugar se involucren, los resultados podrán llegar a ser mejores, esto debido a que todos los trabajadores se sentirán parte del cambio y su sentido de compromiso será mayor.

Al momento de plantear una metodología, esta debe ser sencilla, bien estructurada y ordenada ya que estas características juegan un papel importante para tener éxito en su aplicación. En línea con estas consideraciones, la herramienta guía aquí presentada se considera adecuada ya que demostró ser fácil y con una estructura definida, lo cual fue indispensable para su implementación por parte de los trabajadores del laboratorio.

A la luz de los resultados encontrados mediante la aplicación de la herramienta en el laboratorio, se puede observar que es efectiva para la mejora de procesos dentro de laboratorios de educación superior, pues genera reducciones en tiempo de al menos el 
$50 \%$ en actividades de registro y préstamo, y más del $90 \%$ en tiempos de búsqueda. Además de una reducción importante de espacio y uso de recursos como el papel y la energía, que a pesar de no ser cuantificados, fueron notados por todos los usuarios y trabajadores del lugar.

También cabe resaltar que esta metodología permite el reconocimiento de todas las máquinas y herramientas de los laboratorios con el objetivo de mantener un inventario y programación de mantenimientos actualizados.

En general, el éxito en la aplicación de esta herramienta guía dependerá del compromiso de los involucrados, el buen uso de las herramientas Lean, en especial el Kaizen, y el seguimiento sistemático y estricto del procedimiento descrito.

La aplicación aquí ejecutada fue realizada para un proyecto de mediano plazo, razón por la cual queda abierta la posibilidad de evaluar sus resultados de un proyecto a largo plazo.

\section{REFERENCIAS}

[1] N. Bhuiyan y A. Baghel, "An overview of continuous improvement: from the past to the present”, Manag. Decis., vol. 43, n.․ 5, pp. 761-771, jun. 2005. DOI: 10.1108/00251740510597761

[2] T. Waterbury, "Learning from the pioneers", Int. J. Qual. Reliab. Manag., vol. 32, n. 9 , pp. 934-950, oct. 2015. DOI: 10.1108/IJQRM-08-2014-0125

[3] P. Hines, M. Holweg, y N. Rich, "Learning to evolve”, Int. J. Oper. Prod. Manag., vol. 24, n. ${ }^{\circ}$ 10, pp. 994-1011, oct. 2004. DOI: 10.1108/01443570410558049

[4] M. Holweg, “The genealogy of Lean production”, J. Oper. Manag., vol. 25, n. ${ }^{\circ}$ 2, pp. 420-437, mar. 2007. DOI: 10.1016/j.jom.2006.04.001

[5] M. Espejo y J. Moyano, "Lean production: estado actual y desafíos futuros de la investigación,” Investig. Eur. Dir. y Econ. la Empres., vol. 13, n. ${ }^{\circ}$ 2, pp. 179-202, 2007.

[6] S. Vukadinovic, M. Djapan, y I. Macuzic, "Education for Lean \& Lean for education: A literature review”, Int. J. Qual. Res., vol. 11, n. ${ }^{\circ}$ 1, pp. 35-50, 2017. DOI: 10.18421/IJQR11.01-03

[7] W. K. Balzer, Lean Higher Education Increasing the Value and Performance of University Processes. New York: CRC Press, 2010.

[8] S. Knowles I. Barnes, "Lean laboratories: laboratory medicine needs to learn from other industries how to deliver more for less”, J. Clin. Pathol., vol. 66, n. ${ }^{\circ}$ 8, pp. 635-637, ag. 2013. DOI: 10.1136/jclinpath-2013-201624

[9] M. Vijayshri, J. S.B., y T. D.J., “Applicability of Lean Principles for Testing Laboratories”, Int. J. Sci. Technol. Eng., vol. 3, n. ${ }^{\circ}$ 6, pp. 276-279, 2016.

[10] S. Sreedharan y F. Liou, "Can Lean manufacturing be applied to university laboratories?", ASEE Annu. Conf. Expo. Conf. Proc., 2007. 
[11] P. M. Ramos, "Lean Manufacturing and its application in the laboratory of Häme University of Applied Sciences (HAMK),”, Universidad de Valladolid. Escuela de Ingenierías Industriales, 2017.

[12] H. R. Rizvi, "Application of Lean-Six Sigma Approach in a Laboratory Experimental Case Study”, Int. J. Lean Think., vol. 4, pp. 1-13, 2013.

[13] P. James. (2013). "University labs: waste not, want not | Higher Education Network | The Guardian”. [Internet]. Disponible en: https://www.theguardian.com/higher-education-network/ blog/2013/may/01/university-science-laboratories-efficiency-funding

[14] C. Oberhausen y P. Plapper, "Value stream management in the Lean manufacturing laboratory”, Procedia CIRP, vol. 32, pp. 144-149, 2015. DOI: 10.1016/j.procir.2015.02.087

[15] J. Bhamu y K. Singh Sangwan, "Lean manufacturing: literature review and research issues", Int. J. Oper. Prod. Manag., vol. 34, n. ㄱ, pp. 876-940, 2014. DOI: 10.1108/IJOPM-08-2012-0315

[16] N. V. K. Jasti y R. Kodali, “Lean production: literature review and trends”, Int. J. Prod. Res., vol. 53, n. ${ }^{\circ}$, pp. 867-885, febr. 2015. DOI: 10.1080/00207543.2014.937508

[17] S. Wood. (2006). "How LIMS Facilitates Lean Manufacturing Processes in the Laboratory," American Laboratory. [Internet]. Disponible en: https://www.environmental-expert.com/ Files/9134/articles/11382/AL-Wood-Reprint.pdf

[18] G. Halwachs-Baumann, "Concepts for Lean Laboratory Organization”, J. Med. Biochem., vol. 29, n. ${ }^{\circ}$, pp. 330-338, jun. 2010. DOI: 10.2478/v10011-010-0036-5

[19] Dundas, N. E., Ziadie, M. S., Revell, P. A., Evangeline Brock, Midori Mitui, N. Kristine Leos y Beverly B. Rogers, A Lean Laboratory. The Journal of Molecular Diagnostics, vol. 13, n 2, pp. 175-179, 2011. DOI: 10.1016/j.jmoldx.2010.09.003

[20] M. Herasuta, “A 'Lean' Laboratory”, Lab. Med., vol. 38, n. 3, pp. 143-144, mzo. 2007. DOI: 10.1309/KPVW7AJRQWAA45W0

[21] J. R. Jadhav, S. S. Mantha y S. B. Rane, "Development of framework for sustainable Lean implementation: an ISM approach”, J. Ind. Eng. Int., vol. 10, n. 3, p. 72, 2014. DOI: 10.1007/ s40092-014-0072-8

[22] S. Mostafa, J. Dumrak y H. Soltan, “A framework for Lean manufacturing implementation”, Prod. Manuf. Res., vol. 1, n. ${ }^{\circ}$ 1, pp. 44-64, 2013. DOI: 10.1080/21693277.2013.862159

[23] P. Yadav, B. Nepal, P. Goel, R. Jain y R. P. Mohanty, "Implementation of Lean Manufacturing Principles in Auto Industry”, Ind. Eng. Lett., vol. 1, n. ${ }^{\circ}$ 1, pp. 56-61, 2011. DOI: 10.1504/ IJSOM.2010.032916

[24] A. M. N. Rose, B. M. Deros y M. N. A. Rahman, "Development of framework for Lean manufacturing implementation in SMEs", The 11th Asia Pacific Industrial Engineering and Management Systems Conference, pp. 7-10, 2010.

[25] T. L. E. Institute, Lean Lexicon, 4th ed. Cambrige MA: The Lean Enterprise Institute Inc., 2008. 
[26] J. Sisson y A. Elshennawy, "Achieving success with Lean: An analysis of key factors in Lean transformation at Toyota and beyond", Int. J. Lean Six Sigma, vol. 6, n. ㄱ 3, pp. 263-280, 2015. DOI: 10.1108/IJLSS-07-2014-0024

[27] J. R. Langabeer, J. L. DelliFraine, J. Heineke y I. Abbass, "Implementation of Lean and Six Sigma quality initiatives in hospitals: A goal theoretic perspective," Oper. Manag. Res., vol. 2, n. ${ }^{\circ}$, pp. 13-27, 2009. DOI: 10.1007/s12063-009-0021-7

[28] T. P. Joseph, "Design a Lean laboratory layout”, MLO. Med. Lab. Obs., vol. 38, n. ${ }^{\circ}$ 2, pp. 24-6; pp. 28-9; p. 31, febr. 2006.

[29] T. P. Joseph, "Design of Lean work cells: a Lean lab layout (part II)", MLO. Med. Lab. Obs., vol. 38 , n. ${ }^{\circ} 8$, pp. 24-26; p. 8; pp. 31-2, ag. 2006.

[30] M. Graban, "Riverside Medical Center puts Lean in the laboratory," SME Lean Manuf., pp. 53-57, 2007.

[31] S. S. Raab, D. Grzybicki, L. Condel, W. Stewart, B. Turcsanyi y L. Mahood, "Effect of Lean method implementation in the histopathology section of an anatomical pathology laboratory," J. Clin. Pathol., vol. 61, n. ${ }^{\circ}$ 11, pp. 1193-1199, nov. 2008. DOI: 10.1136/jep.2007.051326

[32] M. Cankovic, R. Varney, L. Whiteley, R. Brown, R. D’Angelo, D. Chitale y R. Zarbo, “The Henry Ford Production System: LEAN Process Redesign Improves Service in the Molecular Diagnostic Laboratory”, J. Mol. Diagnostics, vol. 11, n. 5 , pp. 390-399, sept. 2009. DOI: 10.2353/jmoldx.2009.090002

[33] D. Villa, "Automation, Lean, Six Sigma: Synergies for Improving Laboratory Efficiency", J. Med. Biochem., vol. 29, n. ${ }^{\circ}$ 4, pp. 339-348, jun. 2010. DOI: 10.2478/v10011-010-0038-3

[34] K. Lehmann. (2011). “Lean Laboratory Design.Case Study: Catholic Medical Center, Manchester New Hampshire”, Leica Biosystems. [Internet]. Disponible en: https://drp8p5tqcb2p5. cloudfront.net/fileadmin/downloads_lbs/Leica PELORIS/Testimonials/95.10651_rev_a_ Lean_Laboratory_Design.pdf

[35] B. Das, "Validation Protocol: First Step of a Lean-Total Quality Management Principle in a New Laboratory Set-up in a Tertiary Care Hospital in India”, Indian J. Clin. Biochem., vol. 26, n. ${ }^{\circ}$ 3, pp. 235-243, jul. 2011. DOI: 10.1007/s12291-011-0110-x

[36] J. P. Campos. (2012). "Lean lab in action," MLO: medical laboratory observer. [Internet]. Disponible en: https://www.mlo-online.com/Lean-lab-in-action.php

[37] L. M. Yerian, J. A. Seestadt, E. R. Gomez y K. K. Marchant, "A Collaborative Approach to Lean Laboratory Workstation Design Reduces Wasted Technologist Travel”, Am. J. Clin. Pathol., vol. 138, n. ${ }^{\circ}$ 2, pp. 273-280, ag. 2012. DOI: 10.1309/AJCPE0PI2ENWYWMU

[38] P. S. Mitchell, J. N. Mandrekar y J. D. C. Yao, "Adoption of Lean Principles in a High-Volume Molecular Diagnostic Microbiology Laboratory”, J. Clin. Microbiol., vol. 52, n. 7, pp. 26892693, jul. 2014. DOI: 10.1128/JCM.00430-14 
[39] R. Miniati, F. Frosini, G. Cecconi, F. Dori, E. Ladanza, S. Vezzosi, M. Curiardi y A. Belardinelli, "Experience of Lean Six Sigma Quality Approach to Hospital Laboratory Services", 6th Eur. Conf. Int. Fed. Med. Biol. Eng., vol. 45, pp. 609-612, 2015. 